\title{
A NOTE ON CARTAN-EILENBERG GORENSTEIN CATEGORIES
}

\author{
Bo Lu*, Wei Ren and Zhongkui LiU
}

\begin{abstract}
In this article, we investigate the stability of Cartan-Eilenberg Gorenstein categories. To this end, we introduce and study the concept of two-degree Cartan-Eilenberg $\mathcal{W}$-Gorenstein complexes. We prove that a complex $C$ is two-degree Cartan-Eilenberg $\mathcal{W}$-Gorenstein if and only if $C$ is Cartan-Eilenberg $\mathcal{W}$-Gorenstein. As applications, we show that a complex $C$ is two-degree C-E Gorenstein projective if and only if $C$ is C-E Gorenstein projective. Moreover, we obtain similar results for some known modules such as $\Omega$-Gorenstein projective modules and V-Gorenstein projective modules.
\end{abstract}

\section{Introduction}

A generalization of classical homological algebra is now called Gorenstein homological algebra, which has been developed to an advanced level, see for example $[1,5,6,8,9,10,11,13,16,19,23,24]$. In particular, Sather-Wagstaff et al. in [23] studied the stability of Gorenstein categories. It was shown that over a commutative ring $R$, iterating the process used to define Gorenstein projectove modules exactly leads to the Gorenstein projective modules, i.e., given an exact sequence of Gorenstein projective $R$-modules

$$
G=: \cdots \rightarrow G_{1} \stackrel{\delta_{1}^{G}}{\rightarrow} G_{0} \stackrel{\delta_{0}^{G}}{\rightarrow} G_{-1} \stackrel{\delta_{-1}^{G}}{\rightarrow} \cdots
$$

such that $G$ is $\operatorname{Hom}_{R}(-, H)$ and $\operatorname{Hom}_{R}(H,-)$ exact for each Gorenstein projective $R$-module $H$, the module $\operatorname{Ker}\left(\delta_{0}^{G}\right)$ is Gorenstein projective.

For the rest of the paper, we will use the abbreviation C-E for CartanEilenberg.

Recall that $P$ is a C-E projective complex if $P, \mathrm{Z}(P), \mathrm{B}(P)$ and $\mathrm{H}(P)$ are complexes of projective modules, where $\mathrm{Z}(P), \mathrm{B}(P)$ and $\mathrm{H}(P)$ denote the cycle complex, boundary complex and homology complex of $P$ respectively. Similarly,

2010 Mathematics Subject Classification. 18G10; 18G35; $18 \mathrm{G} 15$.

Key words and phrases. Self-orthogonal class; (two-degree) Cartan-Eilenberg Gorenstein complex; Gorenstein module; semidualizing module.

* Corresponding author.

This research was supported by National Natural Science Foundation of China (No. 11201377, 11261050).

Received October 1, 2013; revised August 29, 2014. 
C-E injective complexes are defined. These complexes have origin in [4] to give the definitions of projective and injective resolutions of a complex of modules.

Triangulated categories were introduced by Grothendieck and Verdier in the early sixties as the proper framework for doing homological algebra in an abelian category. Since triangulated categories have found important applications in algebraic geometry, stable homotopy theory, and representation theory. Examples for this can be found in duality theory (Hartshorne [15] and Iversen [17]) or in the fundamental work on preverse sheaves by Bernstein, Beilinson and Deligne [2].

In [3], Beligiannis introduced projective and injective objects in triangulated categories, i.e., so called $\xi$-projective objects and $\xi$-projective objects, where $\xi$ is a proper class of triangulated categories. In general it is not so easy to find a proper class $\xi$ of triangles in a triangulated category having enough $\xi$-projectives or $\xi$-injectives. Take $\mathcal{C}$ to be the unbounded homotopy category of complexes of modules. Then C-E projective complexes (resp., C-E injective complexes) form the relative projective objects (resp., the relative injective objects) for a proper class of triangles in $\mathcal{C}$, see [3, Sections 12.4 and 12.5]. Therefore, it is necessary and important to study the C-E projective and injective complexes of modules. In 2011, Enochs studied C-E projective and injective complexes. Also the notion of C-E Gorenstein projective and injective complexes was introduced and studied [7]. A complex $G$ is C-E Gorenstein projective if there is a C-E exact sequence of $\mathrm{C}$-E projective complexes

$$
\mathbf{P}=\cdots \rightarrow P^{-2} \rightarrow P^{-1} \rightarrow P^{0} \rightarrow P^{1} \rightarrow P^{2} \rightarrow \cdots
$$

such that $G \cong \operatorname{Ker}\left(P^{0} \rightarrow P^{1}\right)$ and the functor $\operatorname{Hom}(-, Q)$ leaves $\mathbf{P}$ exact whenever $Q$ is C-E projective. C-E Gorenstein injective complexes are defined dually.

In this paper, we are concered with the stability of C-E Gorenstein categories. To this end, we introduced the notion of two-degree C-E Gorenstein projective complexes. Specially, a complex $C$ is called two-degree C-E Gorenstein projective if there exists a $\mathrm{C}$-E exact sequence of $\mathrm{C}$-E Gorenstein projective comlexes

$$
\mathbf{G}=\cdots \rightarrow G^{-2} \rightarrow G^{-1} \rightarrow G^{0} \rightarrow G^{1} \rightarrow G^{2} \rightarrow \cdots
$$

with $C=\operatorname{Ker}\left(G^{0} \rightarrow G^{1}\right)$ such that $\mathbf{G}$ is $\operatorname{Hom}(-, H)$ and $\operatorname{Hom}(H,-)$ exact for each C-E Gorenstein projective complex $H$. Our main results in this note can be stated as follows.

THEOREM 1.1. Let $C$ be a complex and $\mathcal{W}$ a self-orthogonal class of $R$-modules. Then the following statements are equivalent:

(1) $C$ is two-degree $\mathrm{C}-\mathrm{E} \mathcal{W}$-Gorenstein;

(2) $C, \mathrm{Z}(C), \mathrm{B}(C), \mathrm{H}(C), C / \mathrm{Z}(C)$ and $C / \mathrm{B}(C)$ are complexes consisting of $\mathcal{W}$-Gorenstein modules;

(3) $C, \mathrm{Z}(C), \mathrm{B}(C)$ and $\mathrm{H}(C)$ are complexes consisting of $\mathcal{W}$-Gorenstein modules;

(4) $C$ is a C-E $\mathcal{W}$-Gorenstein complex. 
If we set $\mathcal{W}=\mathcal{P}$, where $\mathcal{P}$ denotes the category of projective left $R$-modules, as a consequence of Theorem 1.1, we get the following result.

TheOREM 1.2. A complex $C$ is two-degree C-E Gorenstein projective if and only if $C$ is $\mathrm{C}-\mathrm{E}$ Gorenstein projective.

Dually, we have similar results for C-E Gorenstein injective complexes.

Remark 1.3. It is worth noting that the methods are different from SatherWagstaff et al.'s in discussing stability of Gorenstein categories of modules over commutative rings, and we can extend their main result to any ring as a special case in our present framework.

For any $R$-module $M$, we identify $M$ with the complex $\underline{M}$ concentrated in degree zero. Then $\underline{M}$ is (two-degree) C-E Gorenstein projective if and only if $M$ is (two-degree) Gorenstein projective (by Lemma 4.1 and Corollary 4.9). Therefore, as a special case of our present framework, we extend the main result of [23] from commutative rings to any ring.

Note that the classes of projective modules and injective modules are selforthogonal. Therefore, we first consider self-orthogonal class of $R$-modules $\mathcal{W}$. To this end, we define and study two-degree C-E $\mathcal{W}$-Gorenstein complexes. We show in Theorem 3.10 that a complex $C$ is two-degree C-E $\mathcal{W}$-Gorenstein if and only if $C$ is $\mathrm{C}$-E $\mathcal{W}$-Gorenstein. By a different choice of $\mathcal{W}$, we obtain similar results for some known modules such as $C$-Gorenstein modules, $\Omega$-Gorenstein modules and $V$-Gorenstein modules.

\section{Preliminaries}

Throughout this paper, $R$ denotes an associative ring with unity.

A complex

$$
\cdots \stackrel{\delta_{2}}{\rightarrow} C_{1} \stackrel{\delta_{1}}{\rightarrow} C_{0} \stackrel{\delta_{0}}{\rightarrow} C_{-1} \stackrel{\delta_{-1}}{\rightarrow} \cdots
$$

of left $R$-modules will be denoted by $(C, \delta)$ or $C$.

We will use subscripts to distinguish complexes. So if $\left\{C^{i}\right\}_{i \in I}$ is a family of complexes, $C^{i}$ will be

$$
\cdots \stackrel{\delta_{2}}{\rightarrow} C_{1}^{i} \stackrel{\delta_{1}}{\rightarrow} C_{0}^{i} \stackrel{\delta_{0}}{\rightarrow} C_{-1}^{i} \stackrel{\delta_{-1}}{\rightarrow} \cdots
$$

Given a left $R$-module $M$, we use the notation $D^{n}(M)$ to denote the complex

$$
\cdots \rightarrow 0 \rightarrow M \stackrel{\text { id }}{\rightarrow} M \rightarrow 0 \rightarrow \cdots
$$

with $M$ in the $n$th and $(n-1)$ th positions and set $\bar{M}=D^{0}(M)$. We also use the notation $S^{n}(M)$ to denote the complex with $M$ in the $n$th place and 0 in the other places and set $\underline{M}=S^{0}(M)$. 
Given a complex $C$ and an integer $m, \Sigma^{m} C$ denotes the complex such that $\left(\Sigma^{m} C\right)_{l}=C_{l-m}$, and whose boundary operators are $(-1)^{m} \delta_{l-m}$. The $l$ th homology module of $C$ is the module $\mathrm{H}_{l}(C)=\mathrm{Z}_{l}(C) / \mathrm{B}_{l}(C)$ where $\mathrm{Z}_{l}(C)=\operatorname{Ker}\left(\delta_{l}^{C}\right)$ and $\mathrm{B}_{l}(C)=\operatorname{Im}\left(\delta_{l+1}^{C}\right)$. We set $\mathrm{H}^{l}(C)=\mathrm{H}_{-l}(C)$.

Given two complexes $C$ and $D$, we let $\operatorname{Hom}(C, D)$ denote the abelian group of morphisms from $C$ to $D$ and $\operatorname{Ext}^{i}$ for $i \geq 0$ denote the groups we get from the right derived functor of Hom.

A homomorphism $\varphi: C \rightarrow D$ of degree $n$ is a family $\left(\varphi_{i}\right)_{i \in \mathbf{Z}}$ of homomorphisms of $R$-modules $\varphi_{i}: C_{i} \rightarrow D_{n+i}$. The set of all such homomorphisms forms an abelian group which we denote by $\mathcal{H o m}(C, D)_{n}$. This group is clearly isomorphic to $\prod_{i \in \mathbf{Z}} \operatorname{Hom}_{R}\left(C_{i}, D_{n+i}\right)$. We let $\mathcal{H o m}(C, D)$ denote the complex of abelian groups with $n$th component $\mathcal{H o m}(C, D)_{n}$ and boundary operator

$$
\delta_{n}\left(\left(\varphi_{i}\right)_{i \in \mathbf{Z}}\right)=\left(\delta_{n+i}^{D} \varphi_{i}-(-1)^{n} \varphi_{i-1} \delta_{i}^{C}\right)_{i \in \mathbf{Z}}
$$

The following results can be checked easily:

(1) $\operatorname{Hom}(C, D)=\mathrm{Z}_{0}(\mathcal{H o m}(C, D))$.

(2) If $C$ is a complex and $M$ is an $R$-module, then

$$
\begin{aligned}
& \mathcal{H o m}(C, \underline{M})=\cdots \rightarrow \operatorname{Hom}_{R}\left(C_{-1}, M\right) \rightarrow \operatorname{Hom}_{R}\left(C_{0}, M\right) \rightarrow \operatorname{Hom}_{R}\left(C_{1}, M\right) \rightarrow \cdots, \\
& \mathcal{H o m}(\underline{M}, C)=\cdots \rightarrow \operatorname{Hom}_{R}\left(M, C_{1}\right) \rightarrow \operatorname{Hom}_{R}\left(M, C_{0}\right) \rightarrow \operatorname{Hom}_{R}\left(M, C_{-1}\right) \rightarrow \cdots
\end{aligned}
$$

In the following we recall some notions and facts needed in the sequel.

Let $\mathcal{W}$ be a class of $R$-modules. $\quad \mathcal{W}$ is called self-orthogonal if it satisfies the following condition:

$$
\operatorname{Ext}_{R}^{i}\left(W, W^{\prime}\right)=0 \text { for all } W, W^{\prime} \in \mathcal{W} \text { and all } i \geq 1 .
$$

In the following, $\mathcal{W}$ always denotes a self-orthogonal class of $R$-modules which is closed under extensions, finite direct sums and direct summands. Geng and Ding in [13, Remark 2.3] enumerated a variety of interesting examples of selforthogonal classes.

Definition 2.1 ([13, Definition 2.2]). A left $R$-module $M$ is said to be $\mathcal{W}$-Gorenstein if there is an exact sequence

$$
W_{\bullet}=\cdots \rightarrow W_{2} \rightarrow W_{1} \rightarrow W_{0} \rightarrow W_{-1} \rightarrow W_{-2} \rightarrow \cdots
$$

of modules in $\mathcal{W}$ such that $M \cong \operatorname{Ker}\left(W_{0} \rightarrow W_{-1}\right)$ and $W_{\bullet}$ is $\operatorname{Hom}_{R}(-, \mathcal{W})$ and $\operatorname{Hom}_{R}(\mathcal{W},-)$ exact.

If we take $\mathcal{W}=\mathcal{P}(\mathcal{W}=\mathcal{I})$, then $\mathcal{W}$-Gorenstein modules are exactly Gorenstein projective (Gorenstein injective) modules.

Definition 2.2 ([7, Definition 5.3]). A complex of complexes

$$
\cdots \rightarrow C^{-1} \rightarrow C^{0} \rightarrow C^{1} \rightarrow \cdots
$$


is said to be C-E exact if

(1) $\cdots \rightarrow C^{-1} \rightarrow C^{0} \rightarrow C^{1} \rightarrow \cdots$,

(2) $\cdots \rightarrow \mathrm{Z}\left(C^{-1}\right) \rightarrow \mathrm{Z}\left(C^{0}\right) \rightarrow \mathrm{Z}\left(C^{1}\right) \rightarrow \cdots$

(3) $\cdots \rightarrow B\left(C^{-1}\right) \rightarrow \mathrm{B}\left(C^{0}\right) \rightarrow \mathrm{B}\left(C^{1}\right) \rightarrow \cdots$,

(4) $\cdots \rightarrow C^{-1} / \mathrm{Z}\left(C^{-1}\right) \rightarrow C^{0} / \mathrm{Z}\left(C^{0}\right) \rightarrow C^{1} / \mathrm{Z}\left(C^{1}\right) \rightarrow \cdots$,

(5) $\cdots \rightarrow C^{-1} / \mathrm{B}\left(C^{-1}\right) \rightarrow C^{0} / \mathrm{B}\left(C^{0}\right) \rightarrow C^{1} / \mathrm{B}\left(C^{1}\right) \rightarrow \cdots$,

(6) $\cdots \rightarrow \mathrm{H}\left(C^{-1}\right) \rightarrow \mathrm{H}\left(C^{0}\right) \rightarrow \mathrm{H}\left(C^{1}\right) \rightarrow \cdots$

are all exact.

Definition 2.3. A complex $X$ is said to be a C-E $\mathcal{W}$ complex if $X, \mathrm{Z}(X)$, $\mathrm{B}(X)$ and $\mathrm{H}(X)$ are complexes each of whose terms belongs to $\mathcal{W}$.

Definition 2.4. A complex $G$ is said to be C-E $\mathcal{W}$-Gorenstein, if there exists a $\mathrm{C}$-E exact sequence

$$
\mathbf{W}=\cdots \rightarrow W^{-2} \rightarrow W^{-1} \rightarrow W^{0} \rightarrow W^{1} \rightarrow \cdots
$$

such that

(1) each $W^{i}$ is a C-E $\mathcal{W}$ complex;

(2) $G=\operatorname{Ker}\left(W^{0} \rightarrow W^{1}\right)$;

(3) the sequence remains exact when $\operatorname{Hom}(V,-)$ and $\operatorname{Hom}(-, V)$ are applied to it for any C-E $\mathcal{W}$ complex $V$.

And in this case, $\mathbf{W}$ is called a complete C-E $\mathcal{W}$-resolution of $G$.

In what follows, $R$-Mod denotes the category of left $R$-modules, $\mathscr{C}(R)$ denotes the abelian category of complexes of left $R$-modules. We will use $\mathcal{P}$ and $\mathcal{I}$ to denote the category of projective left $R$-modules and the category of injective left $R$-modules, respectively.

\section{Stability of Cartan-Eilenberg $\mathcal{W}$-Gorenstein categories}

We begin with the following definition.

Definition 3.1. A complex $C$ is said to be two-degree C-E $\mathcal{W}$-Gorenstein if there is a $\mathrm{C}$ - $\mathrm{E}$ exact sequence of $\mathrm{C}$ - $\mathrm{E} \mathcal{W}$-Gorenstein complexes

$$
\mathbf{W}=\cdots \rightarrow W^{-2} \rightarrow W^{-1} \rightarrow W^{0} \rightarrow W^{1} \rightarrow W^{2} \rightarrow \cdots
$$

such that $C \cong \operatorname{Ker}\left(W^{0} \rightarrow W^{1}\right)$ and the functors $\operatorname{Hom}(-, W)$ and $\operatorname{Hom}(W,-)$ leave $\mathbf{W}$ exact whenever $W$ is a C-E $\mathcal{W}$-Gorenstein complex. In this case, $\mathbf{W}$ is called a complete $\mathrm{C}$-E $\mathcal{W}$-Gorenstein resolution of $C$.

It is obvious that any $\mathrm{C}$-E $\mathcal{W}$-Gorenstein complex is two-degree C-E $\mathcal{W}$-Gorenstein.

In this Section, we will investigate the stability of C-E $\mathcal{W}$-Gorenstein categories, where $\mathrm{C}$-E $\mathcal{W}$-Gorenstein categories denote the category of C-E $\mathcal{W}$-Gorenstein complexes and a $\mathrm{C}$-E $\mathcal{W}$-Gorenstein category is said to be stable 
if any two-degree $\mathrm{C}$-E $\mathcal{W}$-Gorenstein complexes are $\mathrm{C}$-E $\mathcal{W}$-Gorenstein. In particular, given a $\mathrm{C}$-E exact sequence of $\mathrm{C}$-E Gorenstein projective complexes

$$
\mathbf{G}=\cdots \rightarrow G^{-1} \rightarrow G^{0} \rightarrow G^{1} \rightarrow \cdots
$$

such that $\mathbf{G}$ is $\operatorname{Hom}(H,-)$ and $\operatorname{Hom}(-, H)$ exact for each C-E Gorenstein projective complex $H$, we will show that the complex $\operatorname{Ker}\left(G^{0} \rightarrow G^{1}\right)$ is C-E Gorenstein projective. Dually, we have similar results for C-E Gorenstein injective complexes.

Now our main aim is to show that two-degree $\mathrm{C}$-E $\mathcal{W}$-Gorenstein complexes are $\mathrm{C}$-E $\mathcal{W}$-Gorenstein. This is done in Theorem 3.10. In the following, we first give the following results.

Lemma 3.2 ([14], Lemma 3.1]). For any $n \in \mathbf{Z}, X \in \mathscr{C}(R)$ and $M \in R$-Mod, we have the following natural isomorphisms:

(1) $\operatorname{Hom}\left(\sum^{n}(\bar{M}), X\right) \cong \operatorname{Hom}_{R}\left(M, X_{n+1}\right)$.

(2) $\operatorname{Hom}\left(\sum^{n}(\underline{M}), X\right) \cong \operatorname{Hom}_{R}\left(M, \mathrm{Z}_{n}(X)\right)$.

(3) $\operatorname{Hom}\left(X, \sum^{n}(\bar{M})\right) \cong \operatorname{Hom}_{R}\left(X_{n}, M\right)$.

(4) $\operatorname{Hom}\left(X, \sum^{n}(\underline{M})\right) \cong \operatorname{Hom}_{R}\left(X_{n} / \mathrm{B}_{n}(X), M\right)$.

Lemma 3.3. (1) The class of $\mathcal{W}$-Gorenstein modules is closed under extensions.

(2) If $M$ is a $\mathcal{W}$-Gorenstein module, then $\operatorname{Ext}_{R}^{1}(M, W)=0$ and $\operatorname{Ext}_{R}^{1}(W, M)$ $=0$ for any $W \in \mathcal{W}$.

Proof. It follows by the definition of $\mathcal{W}$-Gorenstein modules.

Recall that $X$ is called a $\mathcal{W}$ complex [22] if $X$ is acyclic and $Z_{n}(X) \in \mathcal{W}$ for any $n \in \mathbf{Z}$. We will denote the class of $\mathcal{W}$ complexes by $\tilde{\mathcal{W}}$.

In [22, Sect. 5.1], the author defined $\tilde{\mathcal{W}}$-Gorenstein complexes, similar to the definition of $\mathcal{W}$-Gorenstein module, by replacing the modules in $\mathcal{W}$ with complexes in $\tilde{\mathcal{W}}$. It is proved that:

Lemma 3.4 ([22, Theorem A]). A complex $X$ is $\tilde{\mathcal{W}}$-Gorenstein if and only if $X_{n}$ are $\mathcal{W}$-Gorenstein modules for all $n \in \mathbf{Z}$.

Proposition 3.5. $\quad X$ is a C-E $\mathcal{W}$ complex if and only if $X$ can be divided into direct sums $X=X^{\prime} \oplus X^{\prime \prime}$ where $X^{\prime} \in \tilde{\mathcal{W}}$ and $X^{\prime \prime}$ is a graded module with all components in $\mathcal{W}$.

Proof. Since $X^{\prime} \in \tilde{\mathcal{W}}$ is acyclic, $\mathrm{B}_{n}\left(X^{\prime}\right)=\mathrm{Z}_{n}\left(X^{\prime}\right) \in \mathcal{W}, \mathrm{H}_{n}\left(X^{\prime}\right)=0$ for all $n \in \mathbf{Z}$. Then $X^{\prime}$ is a C-E $\mathcal{W}$ complex. It is easy to see $X^{\prime \prime}$ is a C-E $\mathcal{W}$ complex. Then such direct sum is a $\mathrm{C}-\mathrm{E} \mathcal{W}$ complex.

Conversely, suppose that $X$ is a C-E $\mathcal{W}$ complex. We have the exact sequences of $R$-modules

$$
0 \rightarrow \mathrm{B}_{n}(X) \rightarrow \mathrm{Z}_{n}(X) \rightarrow \mathrm{H}_{n}(X) \rightarrow 0, \quad 0 \rightarrow \mathrm{Z}_{n}(X) \rightarrow X_{n} \rightarrow \mathrm{B}_{n-1}(X) \rightarrow 0 .
$$


Since $\mathrm{Z}_{n}(X), \mathrm{B}_{n}(X), \mathrm{H}_{n}(X) \in \mathcal{W}$ for all $n \in \mathbf{Z}$, each sequence splits. This allows us to write $X_{n}=\mathrm{B}_{n}(X) \oplus \mathrm{H}_{n}(X) \oplus \mathrm{B}_{n-1}(X)$. Then

$$
d_{n}: X_{n}=\mathrm{B}_{n}(X) \oplus \mathrm{H}_{n}(X) \oplus \mathrm{B}_{n-1}(X) \rightarrow X_{n-1}=\mathrm{B}_{n-1}(X) \oplus \mathrm{H}_{n-1}(X) \oplus \mathrm{B}_{n-2}(X)
$$

is the map $(x, y, z) \rightarrow(z, 0,0)$. Let $\quad X^{\prime}=\bigoplus_{n \in \mathbf{Z}} D^{n}\left(\mathbf{B}_{n-1}(X)\right)$ and $X^{\prime \prime}=$ $\oplus_{n \in \mathbf{Z}} S^{n}\left(\mathrm{H}_{n}(X)\right)$. Then $X=X^{\prime} \oplus X^{\prime \prime}$, and we obtain the desired direct sum decomposition.

LEMMA 3.6. For a complex $G$, the following are equivalent:

(1) $G$ is a C-E $\mathcal{W}$-Gorenstein complex;

(2) $G, \mathrm{Z}(G), \mathrm{B}(G)$ and $\mathrm{H}(G)$ are complexes consisting of $\mathcal{W}$-Gorenstein modules;

(3) $G, \mathrm{Z}(G), \mathrm{B}(G)$ and $\mathrm{H}(G)$ are $\tilde{\mathcal{W}}$-Gorenstein complexes. modules.

In this case, $G / \mathrm{Z}(G)$ and $G / \mathrm{B}(G)$ are complexes consisting of $\mathcal{W}$-Gorenstein

Proof. We start by noting that $(2) \Leftrightarrow(3)$ is immediate from [22, Theorem A]. $(1) \Rightarrow(2)$. Suppose that

$$
\mathbf{W}=\cdots \rightarrow W^{1} \rightarrow W^{0} \rightarrow W^{-1} \rightarrow W^{-2} \rightarrow \cdots
$$

is a complete $\mathrm{C}$-E $\mathcal{W}$-resolution such that $G=\operatorname{Ker}\left(W^{-1} \rightarrow W^{-2}\right)$. Then, there is an exact sequence of modules in $\mathcal{W}$

$$
\cdots \rightarrow W_{n}^{1} \rightarrow W_{n}^{0} \rightarrow W_{n}^{-1} \rightarrow W_{n}^{-2} \rightarrow \cdots
$$

such that $G_{n}=\operatorname{Ker}\left(W_{n}^{-1} \rightarrow W_{n}^{-2}\right)$ for all $n \in \mathbf{Z}$. For any module $M \in \mathcal{W}$, $\sum^{n}(\bar{M})$ is a C-E $\mathcal{W}$ complex for all $n \in \mathbf{Z}$. It follows from Lemma 3.2 that there is a natural isomorphism $\operatorname{Hom}\left(\sum^{n}(\bar{M}), W^{i}\right) \cong \operatorname{Hom}_{R}\left(M, W_{n}^{i}\right)$ for all $i \in \mathbf{Z}$. Then the following commutative diagram with the first row exact

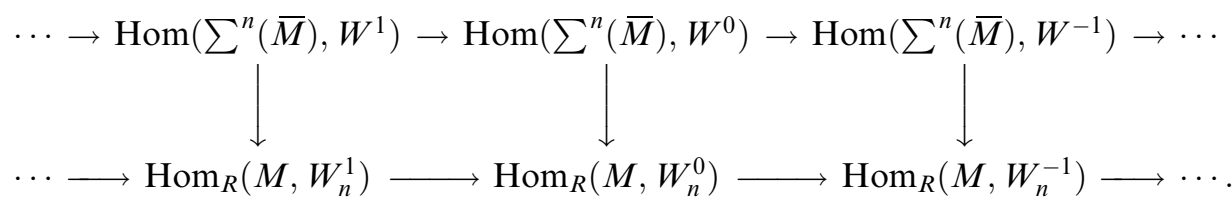

will yield that the lower row is exact. Similarly, there is a natural isomorphism $\operatorname{Hom}\left(W^{i}, \sum^{n}(\bar{M})\right) \cong \operatorname{Hom}_{R}\left(W_{n}^{i}, M\right)$ for all $i \in \mathbf{Z}$. By applying $\operatorname{Hom}\left(-, \sum^{n+1}(\bar{M})\right)$ to the sequence $\mathbf{W}$, we get an exact sequence

$$
\cdots \rightarrow \operatorname{Hom}_{R}\left(W_{n}^{-1}, M\right) \rightarrow \operatorname{Hom}_{R}\left(W_{n}^{0}, M\right) \rightarrow \operatorname{Hom}_{R}\left(W_{n}^{1}, M\right) \rightarrow \cdots .
$$

Hence, $G_{n}$ is a $\mathcal{W}$-Gorenstein module.

For any $n \in \mathbf{Z}$, consider the sequence

$$
\mathrm{Z}_{n}(\mathbf{W})=\cdots \rightarrow \mathrm{Z}_{n}\left(W^{2}\right) \rightarrow \mathrm{Z}_{n}\left(W^{1}\right) \rightarrow \mathrm{Z}_{n}\left(W^{0}\right) \rightarrow \mathrm{Z}_{n}\left(W^{-1}\right) \rightarrow \cdots
$$

It is exact (as $\mathbf{W}$ is $\mathrm{C}$-E exact) with each module $Z_{n}\left(W^{i}\right) \in \mathcal{W}$ for all $i \in \mathbf{Z}$. Note that $Z_{n}(G)=\operatorname{Ker}\left(Z_{n}\left(W^{-1}\right) \rightarrow Z_{n}\left(W^{-2}\right)\right)$. In order to prove $Z_{n}(G)$ is a 
$\mathcal{W}$-Gorenstein module, it suffices to prove that $\mathrm{Z}_{n}(\mathbf{W})$ is both $\operatorname{Hom}_{R}(\mathcal{W},-)$ exact and $\operatorname{Hom}_{R}(-, \mathcal{W})$ exact.

For any module $M \in \mathcal{W}, \sum^{n}(\underline{M})$ is a C-E $\mathcal{W}$ complex for all $n \in \mathbf{Z}$, and there is a natural isomorphism $\operatorname{Hom}\left(\sum^{n}(\underline{M}), W^{i}\right) \cong \operatorname{Hom}_{R}\left(M, Z_{n}\left(W^{i}\right)\right)$ for all $i \in \mathbf{Z}$. By applying $\operatorname{Hom}\left(\sum^{n}(\underline{M}),-\right)$ to the sequence $\mathbf{W}$, an argument similar to the above shows that $\operatorname{Hom}_{R}\left(M, Z_{n}(\mathbf{W})\right)$ is exact.

Since $\mathrm{H}_{n}\left(W^{i}\right)=\mathrm{Z}_{n}\left(W^{i}\right) / \mathrm{B}_{n}\left(W^{i}\right), \quad \mathrm{B}_{n-1}\left(W^{i}\right) \cong W_{n}^{i} / \mathrm{Z}_{n}\left(W^{i}\right)$ are modules in $\mathcal{W}$, the exact sequence $0 \rightarrow \mathrm{H}_{n}\left(W^{i}\right) \rightarrow W_{n}^{i} / \mathrm{B}_{n}\left(W^{i}\right) \rightarrow \mathrm{B}_{n-1}\left(W^{i}\right) \rightarrow 0$ yields that the module $W_{n}^{i} / \mathrm{B}_{n}\left(W^{i}\right)$ belongs to $\mathcal{W}$. Consider the exact sequence of complexes

$$
0 \rightarrow \mathrm{B}_{n}(\mathbf{W}) \rightarrow \mathbf{W}_{n} \rightarrow \mathbf{W}_{n} / \mathrm{B}_{n}(\mathbf{W}) \rightarrow 0
$$

where

$$
\mathrm{B}_{n}(\mathbf{W})=\cdots \rightarrow \mathrm{B}_{n}\left(W^{2}\right) \rightarrow \mathrm{B}_{n}\left(W^{1}\right) \rightarrow \mathrm{B}_{n}\left(W^{0}\right) \rightarrow \mathrm{B}_{n}\left(W^{-1}\right) \rightarrow \cdots .
$$

The sequence is split exact at modules levels, so we have an exact sequence of complexes of $\mathbf{Z}$-modules

$$
0 \rightarrow \operatorname{Hom}_{R}\left(\mathbf{W}_{n} / \mathrm{B}_{n}(\mathbf{W}), M\right) \rightarrow \operatorname{Hom}_{R}\left(\mathbf{W}_{n}, M\right) \rightarrow \operatorname{Hom}_{R}\left(\mathrm{~B}_{n}(\mathbf{W}), M\right) \rightarrow 0 .
$$

Note that $\operatorname{Hom}_{R}\left(\mathbf{W}_{n}, M\right)$ is exact. Also, by applying $\operatorname{Hom}\left(-, \sum^{n}(\underline{M})\right)$ to the sequence $\mathbf{W}$, we get that $\operatorname{Hom}_{R}\left(\mathbf{W}_{n} / \mathbf{B}_{n}(\mathbf{W}), M\right)$ is exact by an argument analogous to the above. Thus $\operatorname{Hom}_{R}\left(\mathbf{B}_{n}(\mathbf{W}), M\right)$ is exact. Similarly, the exact sequence of complexes

$$
0 \rightarrow \mathrm{Z}_{n}(\mathbf{W}) \rightarrow \mathbf{W}_{n} \rightarrow \mathrm{B}_{n-1}(\mathbf{W}) \rightarrow 0
$$

yields an exact sequence of complexes of $\mathbf{Z}$-modules

$$
0 \rightarrow \operatorname{Hom}_{R}\left(\mathbf{B}_{n-1}(\mathbf{W}), M\right) \rightarrow \operatorname{Hom}_{R}\left(\mathbf{W}_{n}, M\right) \rightarrow \operatorname{Hom}_{R}\left(\mathbf{Z}_{n}(\mathbf{W}), M\right) \rightarrow 0,
$$

where $\operatorname{Hom}_{R}\left(\mathbf{B}_{n-1}(\mathbf{W}), M\right)$ and $\operatorname{Hom}_{R}\left(\mathbf{W}_{n}, M\right)$ are exact. So $\operatorname{Hom}_{R}\left(Z_{n}(\mathbf{W}), M\right)$ is exact. This implies that $\mathrm{Z}_{n}(G)$ is a $\mathcal{W}$-Gorenstein module.

There is an exact sequence of modules $0 \rightarrow \mathrm{Z}_{n}(G) \rightarrow G_{n} \rightarrow \mathrm{B}_{n-1}(G) \rightarrow 0$ for all $n \in \mathbf{Z}$ where $\mathrm{Z}_{n}(G), \quad G_{n}$ are $\mathcal{W}$-Gorenstein modules. As above, $\operatorname{Hom}_{R}\left(\mathrm{~B}_{n-1}(\mathbf{W}), M\right)$ is exact for any $M \in \mathcal{W}$, which implies that $\mathrm{B}_{n-1}(G)=$ $\operatorname{Ker}\left(\mathbf{B}_{n-1}\left(W^{-1}\right) \rightarrow \mathbf{B}_{n-1}\left(W^{-2}\right)\right) \in{ }^{\perp} \mathcal{W}$, where ${ }^{\perp} \mathcal{W}=\left\{N \mid \operatorname{Ext}_{R}^{i}(N, M)=0\right.$ for all $M \in \mathcal{W}$ and $i \geq 1\}$. It follows from [13, Corollary $2.6(1)]$ that $\mathrm{B}_{n-1}(G)$ is $\mathcal{W}$-Gorenstein for all $n \in \mathbf{Z}$. Similarly, from the exact sequence of modules $0 \rightarrow \mathrm{B}_{n}(G) \rightarrow \mathrm{Z}_{n}(G) \rightarrow \mathrm{H}_{n}(G) \rightarrow 0$ we can get that $\mathrm{H}_{n}(G)$ is $\mathcal{W}$-Gorenstein. This completes the proof.

$(2) \Rightarrow(1)$. For any $n \in \mathbf{Z}$, consider the exact sequence of modules

$$
0 \rightarrow \mathrm{B}_{n}(G) \rightarrow \mathrm{Z}_{n}(G) \rightarrow \mathrm{H}_{n}(G) \rightarrow 0,
$$

where $\mathrm{B}_{n}(G)$ and $\mathrm{H}_{n}(G)$ are $\mathcal{W}$-Gorenstein. Suppose $W^{\mathrm{B}_{n}(G)}$ and $W^{\mathrm{H}_{n}(G)}$ are complete $\mathcal{W}$-resolutions of $\mathrm{B}_{n}(G)$ and $\mathrm{H}_{n}(G)$, respectively. By the Horseshoe 
Lemma, we can construct a complete $\mathcal{W}$ resolution of $\mathrm{Z}_{n}(G): W^{\mathrm{Z}_{n}(G)}=$ $W^{\mathrm{B}_{n}(G)} \oplus W^{\mathrm{H}_{n}(G)}$. Similarly, consider the exact sequence of modules

$$
0 \rightarrow \mathrm{Z}_{n}(G) \rightarrow G_{n} \rightarrow \mathrm{B}_{n-1}(G) \rightarrow 0,
$$

and we can construct a complete $\mathcal{W}$-resolution of $G_{n}: W^{G_{n}}=W^{Z_{n}(G)} \oplus W^{\mathrm{B}_{n-1}(G)}$ $=W^{\mathrm{B}_{n}(G)} \oplus W^{\mathrm{H}_{n}(G)} \oplus W^{\mathrm{B}_{n-1}(G)}$. Set $\quad W_{n}^{i}=W_{i}^{\mathrm{B}_{n}(G)} \oplus W_{i}^{\mathrm{H}_{n}(G)} \oplus W_{i}^{\mathrm{B}_{n-1}(G)} \quad$ and $d_{n}^{W^{i}}: W_{n}^{i} \rightarrow W_{n-1}^{i}$ which maps $(x, y, z)$ to $(z, 0,0)$ for all $i, n \in \mathbf{Z}$. Then $\left(W^{i}, d^{W^{i}}\right)$ is a complex such that $G_{n}=\operatorname{Ker}\left(W_{n}^{-1} \rightarrow W_{n}^{-2}\right)$.

It is easily seen that $W^{i}$ is a C-E $\mathcal{W}$ complex for all $i \in \mathbf{Z}$ and $G=$ $\operatorname{Ker}\left(W^{-1} \rightarrow W^{-2}\right)$. For any $n \in \mathbf{Z}, \cdots \rightarrow \mathbf{Z}_{n}\left(W^{1}\right) \rightarrow \mathbf{Z}_{n}\left(W^{0}\right) \rightarrow \mathbf{Z}_{n}\left(W^{-1}\right) \rightarrow \cdots$ is a complete $\mathcal{W}$-resolution of $Z_{n}(G)$, and $\cdots \rightarrow W_{n}^{1} \rightarrow W_{n}^{0} \rightarrow W_{n}^{-1} \rightarrow \cdots$ is a complete $\mathcal{W}$-resolution of $G_{n}$, so they both are exact. Hence, we can get that

$$
\mathbf{W}=\cdots \rightarrow W^{1} \rightarrow W^{0} \rightarrow W^{-1} \rightarrow W^{-2} \rightarrow \cdots
$$

is C-E exact by [7, Lemma 5.2]. It remains to prove that, for any C-E $\mathcal{W}$ complex $V, \mathbf{W}$ is still exact when $\operatorname{Hom}(V,-), \operatorname{Hom}(-, V)$ applied to it.

However, it suffices to prove that the assertion holds when we pick $V$ particularly as $V=\sum^{n}(\bar{M})$ and $V=\sum^{n}(\underline{M})$ for any module $M \in \mathcal{W}$ and all $n \in \mathbf{Z}$ by Proposition 3.5. Note that $\mathbf{W}_{n}, Z_{n}(\mathbf{W})$ and $\mathbf{W}_{n} / \mathbf{B}_{n}(\mathbf{W})$ are complete $\mathcal{W}$-resolutions, hence from Lemma 3.2 the desired result follows.

Lemma 3.7. Let $C$ be an exact complex with $\mathcal{H o m}(\underline{W}, C)$ and $\mathcal{H o m}(C, \underline{W})$ exact for any $W \in \mathcal{W}$. Then $C$ is a complex of $\mathcal{W}$-Gorenstein modules if and only if $\mathbf{Z}_{n}(C)$ is $\mathcal{W}$-Gorenstein in $R$-Mod for all $n \in \mathbf{Z}$.

Proof. $(\Rightarrow)$ Let $C$ be a complex of $\mathcal{W}$-Gorenstein modules. Then it follows from Lemma 3.4 that $C$ is $\tilde{\mathcal{W}}$-Gorenstein. There exists an exact sequence of $\mathcal{W}$ complexes

$$
\mathbf{W}=\cdots \longrightarrow W^{-2} \stackrel{f^{-2}}{\longrightarrow} W^{-1} \stackrel{f^{-1}}{\longrightarrow} W^{0} \stackrel{f^{0}}{\longrightarrow} W^{1} \stackrel{f^{1}}{\longrightarrow} W^{2} \longrightarrow \cdots
$$

with $C=\operatorname{Ker} f^{0}$ such that $\mathbf{W}$ is $\operatorname{Hom}(-, V)$ and $\operatorname{Hom}(V,-)$ exact for any $\mathcal{W}$ complex $V$. We also have $\operatorname{Ker}\left(f^{i}\right)$ is exact for all $i \in \mathbf{Z}$ since $\operatorname{Ker}\left(f^{0}\right)=C$ and $W^{i}$ are exact.

Note that $\operatorname{Ext}^{1}\left(\Sigma^{n}(\underline{R}), X\right)=\mathrm{H}_{n-1}(X)=0$ for any exact complex $X$ and $n \in \mathbf{Z}$. By applying $\operatorname{Hom}\left(\Sigma^{n}(\underline{R}),-\right)$ to the sequence $(\star)$, there is a commutative diagram from Lemma 3.2:

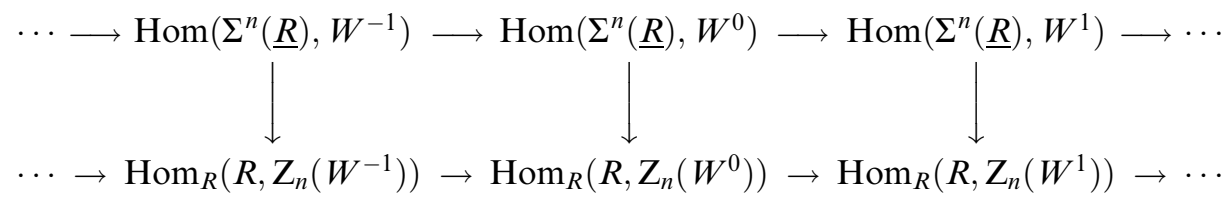


with the upper exact since $\operatorname{Ext}^{1}\left(\Sigma^{n}(\underline{R}), \operatorname{Ker} f^{i}\right)=0$. Then the second row is exact. Thus we have an exact sequence of $\mathcal{W}$ modules

$$
\cdots \rightarrow \mathrm{Z}_{n}\left(W^{-1}\right) \rightarrow \mathrm{Z}_{n}\left(W^{0}\right) \rightarrow \mathrm{Z}_{n}\left(W^{1}\right) \rightarrow \cdots
$$

with $\mathrm{Z}_{n}(C) \cong \operatorname{Ker}\left(\mathrm{Z}_{n}\left(W^{0}\right) \rightarrow \mathrm{Z}_{n}\left(W^{1}\right)\right)$. So we only need to show that $\operatorname{Hom}_{R}(-, W)$ and $\operatorname{Hom}_{R}(W,-)$ leave the sequence $(*)$ exact when $W \in \mathcal{W}$.

Let $W$ be in $\mathcal{W}$ and $g: Z_{n}(C) \rightarrow W$ be a morphism of $R$-modules. Since $\mathcal{H} \operatorname{om}(C, \underline{W})$ is exact, there exists a morphism $f: C_{n} \rightarrow W$ such that the following diagram:

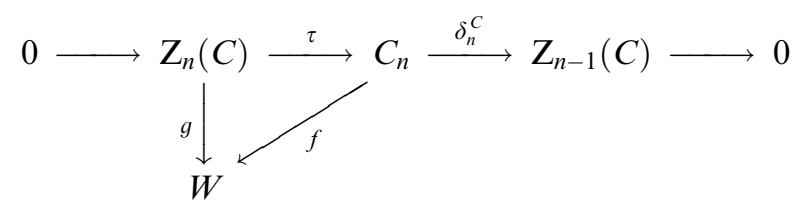

commutes.

Note that $\mathbf{W}$ is $\operatorname{Hom}\left(-, \Sigma^{n} \bar{W}\right)$ exact since $\Sigma^{n}(\bar{W})$ is a $\mathcal{W}$ complex. For the exact sequence $0 \rightarrow C \rightarrow W^{0} \rightarrow \operatorname{Ker} f^{1} \rightarrow 0$, there is an exact sequence $0 \rightarrow$ $\operatorname{Hom}\left(\operatorname{Ker} f^{1}, \Sigma^{n}(\bar{W})\right) \rightarrow \operatorname{Hom}\left(W^{0}, \Sigma^{n}(\bar{W})\right) \rightarrow \operatorname{Hom}\left(C, \Sigma^{n}(\bar{W})\right) \rightarrow 0$. It follows from Lemma 3.2 that $0 \rightarrow \operatorname{Hom}_{R}\left(\left(\operatorname{Ker} f^{1}\right)_{n}, W\right) \rightarrow \operatorname{Hom}\left(W_{n}^{0}, W\right) \rightarrow \operatorname{Hom}\left(C_{n}, W\right)$ $\rightarrow 0$ is exact. Thus, for $f \in \operatorname{Hom}_{R}\left(C_{n}, W\right)$, there exists a morphism $h: W_{n}^{0} \rightarrow W$ such that $f=h \alpha_{n}$. Therefore, we have the following commutative diagram:

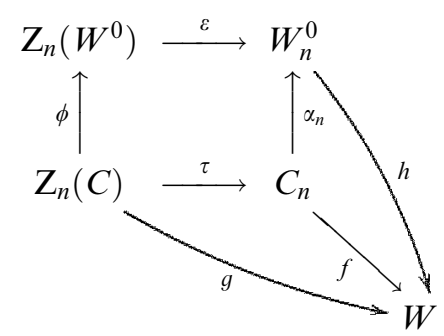

where $\varepsilon: Z_{n}\left(W^{0}\right) \rightarrow W_{n}^{0}$ is the inclusion map, $\phi: Z_{n}(C) \rightarrow Z_{n}\left(W^{0}\right)$ is induced by the morphism $C \rightarrow W^{0}$. And so $g=h \varepsilon \phi$. This implies that

$$
0 \rightarrow \operatorname{Hom}_{R}\left(\mathrm{Z}_{n}\left(\operatorname{Ker} f^{1}\right), W\right) \rightarrow \operatorname{Hom}_{R}\left(\mathbf{Z}_{n}\left(W^{0}\right), W\right) \rightarrow \operatorname{Hom}_{R}\left(\mathbf{Z}_{n}(C), W\right) \rightarrow 0
$$

is exact. Similarly, we can prove that

$$
\begin{aligned}
0 & \rightarrow \operatorname{Hom}_{R}\left(\mathbf{Z}_{n}\left(\operatorname{Ker} f^{i+1}\right), W\right) \rightarrow \operatorname{Hom}_{R}\left(\mathbf{Z}_{n}\left(W^{i}\right), W\right) \\
& \rightarrow \operatorname{Hom}_{R}\left(\mathbf{Z}_{n}\left(\operatorname{Ker} f^{i}\right), W\right) \rightarrow 0
\end{aligned}
$$

is exact. Hence, $\operatorname{Hom}_{R}(-, W)$ leaves the sequence $(*)$ exact. Dually, we can also show that $\operatorname{Hom}_{R}(W,-)$ leaves the sequence $(*)$ exact. This completes the proof. 
$(\Leftarrow)$ Since the sequence

$$
0 \rightarrow \mathrm{Z}_{n+1}(C) \rightarrow C_{n+1} \rightarrow \mathrm{Z}_{n}(C) \rightarrow 0
$$

is exact, $\mathrm{Z}_{n+1}(C)$ and $\mathrm{Z}_{n}(C)$ are $\mathcal{W}$-Gorenstein modules, $C_{n+1}$ is a $\mathcal{W}$-Gorenstein module for all $n \in \mathbf{Z}$ by Lemma 3.3, as desired.

Take $\mathcal{W}=\mathcal{P}$ (respectively, $\mathcal{W}=\mathcal{I}$ ), the subcategory of projective (respectively, injective) $R$-modules. We have the following results.

Corollary 3.8. Let $C$ be an exact complex with $\mathcal{H o m}(C, \underline{P})$ exact for any $P \in \mathcal{P}$. Then $C$ is Gorenstein projective if and only if $\mathrm{Z}_{n}(C)$ is Gorenstein projective in $R$-Mod for all $n \in \mathbf{Z}$.

COROllary 3.9. Let $C$ be an exact complex with $\mathcal{H o m}(\underline{I}, C)$ exact for any $I \in \mathcal{I}$. Then $C$ is Gorenstein injective if and only if $\mathrm{Z}_{n}(C)$ is Gorenstein injective in $R$-Mod for all $n \in \mathbf{Z}$.

THeOREM 3.10. Let $C$ be a complex. Then the following statements are equivalent:

(1) $C$ is two-degree $\mathrm{C}$-E $\mathcal{W}$-Gorenstein;

(2) $C, \mathrm{Z}(C), \mathrm{B}(C), \mathrm{H}(C), C / \mathrm{Z}(C)$ and $C / \mathrm{B}(C)$ are complexes consisting of $\mathcal{W}$-Gorenstein modules;

(3) $C, \mathrm{Z}(C), \mathrm{B}(C)$ and $\mathrm{H}(C)$ are complexes consisting of $\mathcal{W}$-Gorenstein modules;

(4) $C$ is a C-E $\mathcal{W}$-Gorenstein complex.

Proof. $\quad(1) \Rightarrow(2)$. By (1), there exists a C-E exact sequence of C-E $\mathcal{W}$-Gorenstein complexes

$$
\mathbf{W}=\cdots \rightarrow W^{-2} \rightarrow W^{-1} \rightarrow W^{0} \rightarrow W^{1} \rightarrow W^{2} \rightarrow \cdots
$$

with $C=\operatorname{Ker}\left(W^{0} \rightarrow W^{1}\right)$ and such that the functors $\operatorname{Hom}(-, V)$ and $\operatorname{Hom}(V,-)$ leave $\mathbf{W}$ exact whenever $V$ is a $\mathrm{C}$-E $\mathcal{W}$-Gorenstein complex. Then there is an exact sequence of $\mathcal{W}$-Gorenstein modules

$$
\mathbf{W}_{n}=\cdots \rightarrow W_{n}^{-2} \rightarrow W_{n}^{-1} \rightarrow W_{n}^{0} \rightarrow W_{n}^{1} \rightarrow W_{n}^{2} \rightarrow \cdots
$$

with $C_{n}=\operatorname{Ker}\left(W_{n}^{0} \rightarrow W_{n}^{1}\right)$ for all $n \in \mathbf{Z}$ by Lemma 3.6.

Let $W$ be in $\mathcal{W}$. Then $\Sigma^{n-1}(\bar{W})$ and $\Sigma^{n}(\bar{W})$ are C-E $\mathcal{W}$-Gorenstein complexes by Lemma 3.6. We apply the functors $\operatorname{Hom}\left(\Sigma^{n-1}(\bar{W}),-\right)$ and $\operatorname{Hom}\left(-, \Sigma^{n}(\bar{W})\right)$ to the sequence $(\dagger)$, respectively, we get the exact sequences

$$
\cdots \rightarrow \operatorname{Hom}_{R}\left(W, W_{n}^{-1}\right) \rightarrow \operatorname{Hom}_{R}\left(W, W_{n}^{0}\right) \rightarrow \operatorname{Hom}_{R}\left(W, W_{n}^{1}\right) \rightarrow \cdots
$$

and

$$
\cdots \rightarrow \operatorname{Hom}_{R}\left(W_{n}^{1}, W\right) \rightarrow \operatorname{Hom}_{R}\left(W_{n}^{0}, W\right) \rightarrow \operatorname{Hom}_{R}\left(W_{n}^{-1}, W\right) \rightarrow \cdots
$$

for all $n \in \mathbf{Z}$ by Lemma 3.2. And so $C_{n}$ is a $\mathcal{W}$-Gorenstein module for all $n \in \mathbf{Z}$ by Lemma 3.6. 
We also have an exact sequence

$$
\cdots \rightarrow W_{n}^{-1} / \mathrm{Z}_{n}\left(W^{-1}\right) \rightarrow W_{n}^{0} / \mathrm{Z}_{n}\left(W^{0}\right) \rightarrow W_{n}^{1} / \mathrm{Z}_{n}\left(W^{1}\right) \rightarrow \cdots
$$

of $\mathcal{W}$-Gorenstein modules with $C_{n} / Z_{n}(C)=\operatorname{Ker}\left(W_{n}^{0} / Z_{n}\left(W^{0}\right) \rightarrow W_{n}^{1} / Z_{n}\left(W^{1}\right)\right)$ for all $n \in \mathbf{Z}$. We now consider the commutative diagram:

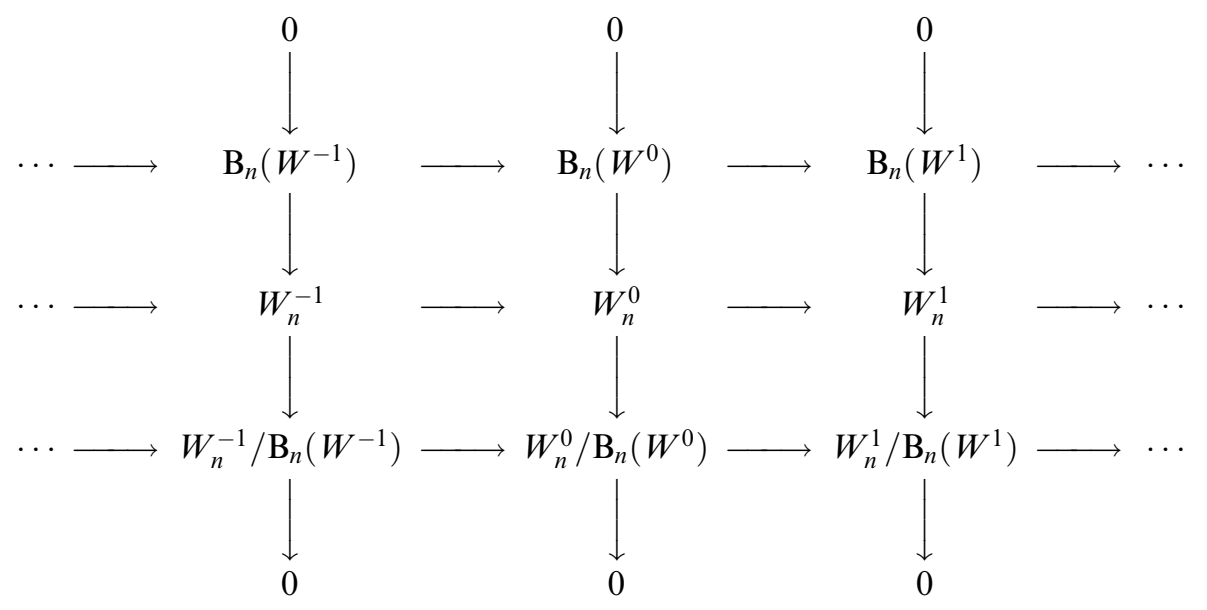

with exact rows and columns. We apply the functor $\operatorname{Hom}_{R}(-, W)$ to the commutative diagram. Since $\operatorname{Ext}_{R}^{1}\left(W_{n}^{i} / \mathrm{B}_{n}\left(W^{i}\right), W\right)=0$ by Lemma 3.3 and Lemma 3.6, we get the commutative diagram:

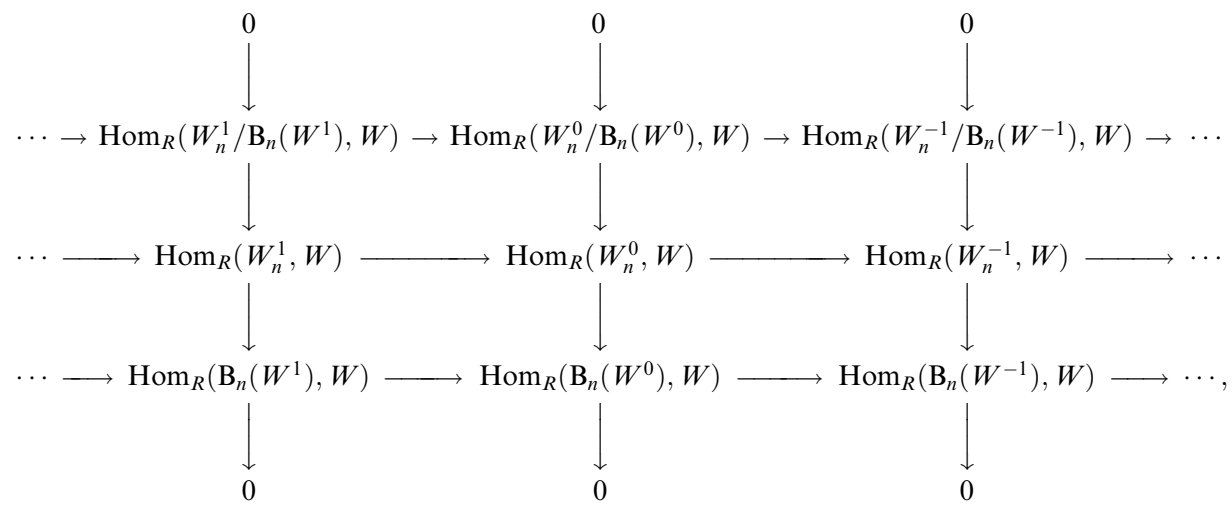

where the first two rows are exact. So the last row is also exact, which means that

$$
\begin{aligned}
\cdots & \rightarrow \operatorname{Hom}_{R}\left(W_{n}^{1} / Z_{n}\left(W^{1}\right), W\right) \rightarrow \operatorname{Hom}_{R}\left(W_{n}^{0} / Z_{n}\left(W^{0}\right), W\right) \\
& \rightarrow \operatorname{Hom}_{R}\left(W_{n}^{-1} / Z_{n}\left(W^{-1}\right), W\right) \rightarrow \cdots
\end{aligned}
$$

is exact since $W_{n}^{i} / \mathrm{Z}_{n}\left(W^{i}\right) \cong \mathrm{B}_{n-1}\left(W_{i}\right)$ for all $i, n \in \mathbf{Z}$. 
On the other hand, we have the commutative diagram:

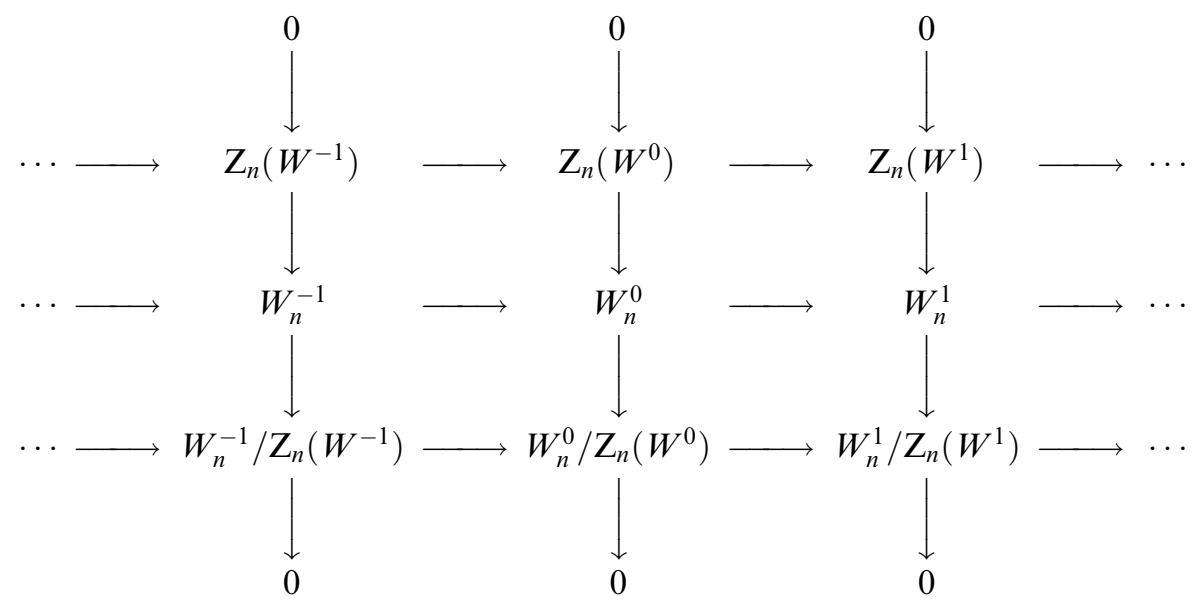

with exact rows and columns. Applying the functor $\operatorname{Hom}_{R}(W,-)$ to this diagram yields the following commutative diagram:

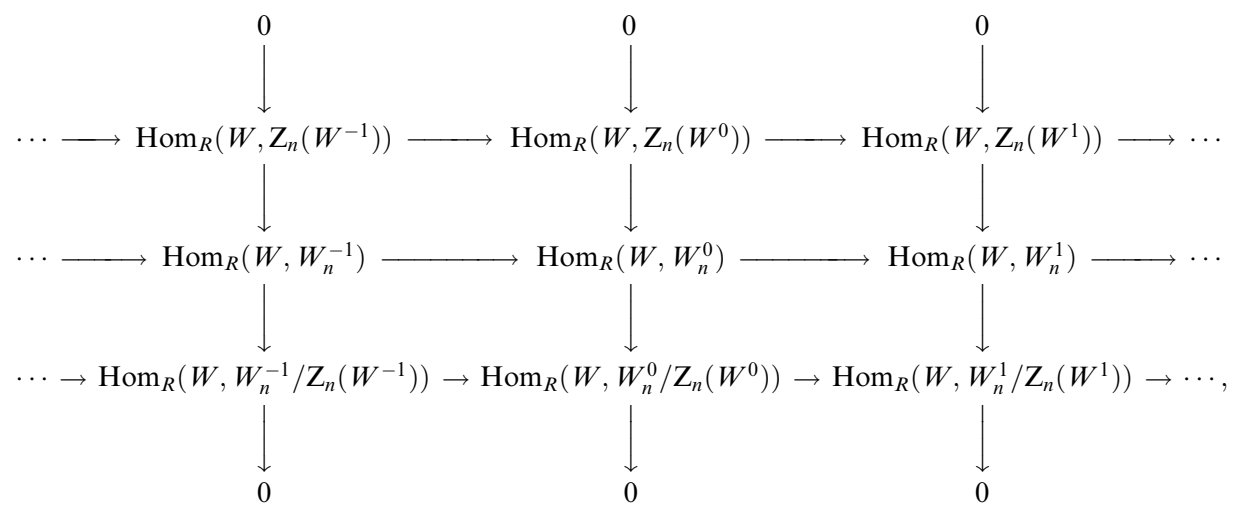

where the first two rows are exact. So the last row is also exact. Therefore,

$$
\mathcal{H o m}\left(\underline{W}, \mathbf{W}_{n} / \mathrm{Z}_{n}(\mathbf{W})\right)
$$

and

$$
\mathcal{H o m}\left(\mathbf{W}_{n} / \mathbf{Z}_{n}(\mathbf{W}), \underline{W}\right)
$$

are exact, where

$$
\mathrm{Z}_{n}(\mathbf{W})=\cdots \rightarrow \mathrm{Z}_{n}\left(W^{-1}\right) \rightarrow \mathrm{Z}_{n}\left(W^{0}\right) \rightarrow \mathrm{Z}_{n}\left(W^{1}\right) \rightarrow \cdots .
$$

Note that $C_{n} / \mathbf{Z}_{n}(C)=\operatorname{Ker}\left(W_{n}^{0} / \mathbf{Z}_{n}\left(W^{0}\right) \rightarrow W_{n}^{1} / \mathbf{Z}_{n}\left(W^{1}\right)\right)$. This implies that $C_{n} / \mathrm{Z}_{n}(C)$ is a $\mathcal{W}$-Gorenstein module for each $n \in \mathbf{Z}$ by Lemma 3.7. 
It is obvious that the sequence

$\cdots \rightarrow \operatorname{Hom}_{R}\left(W, Z_{n}\left(W^{-1}\right)\right) \rightarrow \operatorname{Hom}_{R}\left(W, Z_{n}\left(W^{0}\right)\right) \rightarrow \operatorname{Hom}_{R}\left(W, Z_{n}\left(W^{1}\right)\right) \rightarrow \cdots$.

is exact for all $n \in \mathbf{Z}$.

Moreover, the exact sequence of complexes

$$
0 \rightarrow \mathrm{Z}_{n}(\mathbf{W}) \rightarrow \mathbf{W}_{n} \rightarrow \mathbf{W}_{n} / \mathrm{Z}_{n}(\mathbf{W}) \rightarrow 0
$$

induces exact sequence

$$
0 \rightarrow \mathcal{H} \mathrm{om}\left(\mathbf{W}_{n} / \mathbf{Z}_{n}(\mathbf{W}), \underline{W}\right) \rightarrow \mathcal{H o m}\left(\mathbf{W}_{n}, \underline{W}\right) \rightarrow \mathcal{H o m}\left(\mathbf{Z}_{n}(\mathbf{W}), \underline{W}\right) \rightarrow 0
$$

since $W_{n}^{m} / Z_{n}\left(W^{m}\right)$ is $\mathcal{W}$-Gorenstein in $R$-Mod for all $m, n \in \mathbf{Z}$.

Since $\mathcal{H o m}(\mathbf{W}, \underline{W})$ and $\mathcal{H o m}\left(\mathbf{W} / \mathbf{Z}_{n}(\mathbf{W}), \underline{W}\right)$ are exact, then $\mathcal{H o m}\left(\mathbf{Z}_{n}(\mathbf{W}), \underline{W}\right)$ is exact. Note that $Z_{n}(C)=\operatorname{Ker}\left(Z_{n}\left(W^{0}\right) \rightarrow Z_{n}\left(W^{1}\right)\right)$. Therefore, $Z_{n}(C)$ is a $\mathcal{W}$-Gorenstein module for all $n \in \mathbf{Z}$ by Lemma 3.7.

Similarly, the exactness of

$$
0 \rightarrow \mathrm{B}_{n}(\mathbf{W}) \rightarrow \mathbf{W}_{n} \rightarrow \mathbb{W}_{n} / \mathrm{B}_{n}(\mathbf{W}) \rightarrow 0
$$

gives the exactness of $\mathcal{H o m}\left(\underline{W}, \mathbf{W}_{n} / \mathbf{B}_{n}(\mathbf{W})\right)$.

Applying the functor $\operatorname{Hom}\left(-, \Sigma^{n}(\underline{W})\right)$ to the sequence $(\dagger)$ yields the exact sequence

$$
\begin{aligned}
\cdots & \rightarrow \operatorname{Hom}_{R}\left(W_{n}^{1} / \mathrm{B}_{n}\left(W^{1}\right), W\right) \rightarrow \operatorname{Hom}_{R}\left(W_{n}^{0} / \mathrm{B}_{n}\left(W^{0}\right), W\right) \\
& \rightarrow \operatorname{Hom}_{R}\left(W_{n}^{-1} / \mathrm{B}_{n}\left(W^{-1}\right), W\right) \rightarrow \cdots
\end{aligned}
$$

Note that $C_{n} / \mathrm{B}_{n}(C)=\operatorname{Ker}\left(W_{n}^{0} / \mathrm{B}_{n}\left(W^{0}\right) \rightarrow W_{n}^{1} / \mathrm{B}_{n}\left(W^{1}\right)\right)$. This means that $C_{n} / \mathrm{B}_{n}(C)$ is a $\mathcal{W}$-Gorenstein module for each $n \in \mathbf{Z}$ by Lemma 3.7.

Note that the exactness of

$$
0 \rightarrow \mathrm{B}_{n}(\mathbf{W}) \rightarrow \mathrm{Z}_{n}(\mathbf{W}) \rightarrow \mathrm{H}_{n}(\mathbf{W}) \rightarrow 0
$$

and

$$
0 \rightarrow \mathrm{H}_{n}(\mathbf{W}) \rightarrow \mathbf{W}_{n} / \mathrm{B}_{n}(\mathbf{W}) \rightarrow \mathbf{W}_{n} / \mathrm{Z}_{n}(\mathbf{W}) \rightarrow 0
$$

gives the exactness of $\mathcal{H} \operatorname{om}\left(\underline{W}, \mathrm{H}_{n}(\mathbf{W})\right)$ and $\mathcal{H}$ om $\left(\mathrm{H}_{n}(\mathbf{W}), \underline{W}\right)$. We get $\mathrm{H}_{n}(C)$ are $\mathcal{W}$-Gorenstein modules for all $n \in \mathbf{Z}$ by Lemma 3.7. As desired.

(2) $\Rightarrow(3)$ is trivial.

(3) $\Rightarrow(4)$. It follows by Lemma 3.6.

$(4) \Rightarrow(1)$ is obvious.

\section{Applications}

Lemma 4.1 (Dual version of [7, Theorem 8.5]). For a complex $G$, the following statements are equivalent:

(1) $G$ is C-E Gorenstein projective; modules.

(2) $G / \mathrm{Z}(G)$ and $\mathrm{H}(G)$ are complexes consisting of Gorenstein projective 
In this case, $G, \mathrm{Z}(G), \mathrm{B}(G)$ and $G / \mathrm{B}(G)$ are complexes consisting of Gorenstein projective modules.

In particular, if we set $\mathcal{W}=\mathcal{P}$, then two-degree C-E $\mathcal{W}$-Gorenstein complexes are exactly two-degree C-E Gorenstein projective complexes. We are now in a position to get Theorem 1.2 from the introduction by Lemma 4.1.

Corollary 4.2. A complex $C$ is two-degree C-E Gorenstein projective if and only if $C$ is C-E Gorenstein projective.

Dually, if we set $\mathcal{W}=\mathcal{I}$, then we have:

Corollary 4.3. A complex $C$ is two-degree C-E Gorenstein injective if and only if $C$ is $\mathrm{C}-\mathrm{E}$ Gorenstein injective.

Definition 4.4. A left $R$-module $M$ is said to be two-degree $\mathcal{W}$-Gorenstein if there is an exact sequence of $\mathcal{W}$-Gorenstein modules

$$
W=\cdots \rightarrow W_{2} \rightarrow W_{1} \rightarrow W_{0} \rightarrow W_{-1} \rightarrow W_{-2} \rightarrow \cdots
$$

such that $M \cong \operatorname{Ker}\left(W_{0} \rightarrow W_{-1}\right)$ and the functors $\operatorname{Hom}_{R}(-, V)$ and $\operatorname{Hom}_{R}(V,-)$ leave $W$ exact whenever $V$ is a $\mathcal{W}$-Gorenstein module. In this case, $W$ is called a complete $\mathcal{W}$-Gorenstein resolution of $M$.

Lemma 4.5. Let $M$ be a two-degree $\mathcal{W}$-Gorenstein module. Then $\underline{M}$ is a two-degree C-E $\mathcal{W}$-Gorenstein complex.

Proof. Since $M$ is a two-degree $\mathcal{W}$-Gorenstein module, there exists an exact sequence of $\mathcal{W}$-Gorenstein modules

$$
W=\cdots \rightarrow W_{1} \rightarrow W_{0} \rightarrow W_{-1} \rightarrow \cdots
$$

with $M=\operatorname{Ker}\left(W_{0} \rightarrow W_{-1}\right)$ and such that the functors $\operatorname{Hom}_{R}(-, H)$ and $\operatorname{Hom}_{R}(H,-)$ leave $W$ exact whenever $H$ is a $\mathcal{W}$-Gorenstein module. Then we get a $\mathrm{C}$-E exaxt sequence of $\mathrm{C}$-E $\mathcal{W}$-Gorenstein complexes

$$
\underline{W}=\cdots \rightarrow \underline{W_{1}} \rightarrow \underline{W_{0}} \rightarrow \underline{W_{-1}} \rightarrow \cdots
$$

with $\underline{M}=\operatorname{Ker}\left(\underline{W_{0}} \rightarrow \underline{W_{-1}}\right)$ by Theorem 3.10 .

For any $\mathrm{C}-\overline{\mathrm{E}} \mathcal{W}$-Gorenstein complex $V$, applying the functor $\operatorname{Hom}(V,-)$ to $\underline{W}$ yields the commutative diagram:

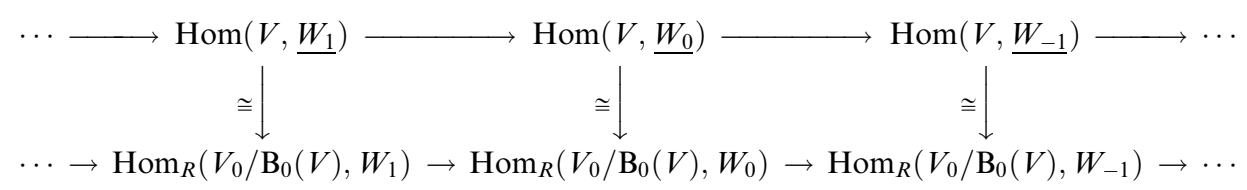


with the second row exact by Theorem 3.10, Lemma 3.2 and Definition 4.4. So the first row is exact.

Dually, if we apply the functor $\operatorname{Hom}(-, V)$ to $\underline{W}$, we also have

$$
\cdots \rightarrow \operatorname{Hom}\left(\underline{W_{-1}}, V\right) \rightarrow \operatorname{Hom}\left(\underline{W_{0}}, V\right) \rightarrow \operatorname{Hom}\left(\underline{W_{1}}, V\right) \rightarrow \cdots
$$

is exact, Therefore, $\underline{M}$ is a two-degree C-E $\mathcal{W}$-Gorenstein complex.

By an argument similar to Lemma 4.5. we have:

Lemma 4.6. Let $M$ be a two-degree $\mathcal{W}$-Gorenstein module. Then $\bar{M}$ is a two-degree $\mathrm{C}$-E $\mathcal{W}$-Gorenstein complex.

COROLlary 4.7. A left R-module $M$ is two-degree $\mathcal{W}$-Gorenstein if and only if $M$ is $\mathcal{W}$-Gorenstein.

Proof. It follows by Theorem 3.10 and Lemma 4.5.

If we take $\mathcal{W}=\mathcal{P}$, by Proposition 4.4, we have the following result which is also the main result of [23].

Corollary 4.8. A left $R$-module $M$ is two-degree Gorenstein projective if and only if $M$ is Gorenstein projective.

Sather-Wagstaff et al. proved in [23] that an $R$-module $M$ is two-degree Gorenstein projective if and only if $M$ is Gorenstein projective over a commutative ring $R$. In this present paper, we obtain the same result over any ring $R$ by different methods.

Theorem 3.10 shows that $C, \mathrm{Z}(C), \mathrm{B}(C), \mathrm{H}(C), C / \mathrm{Z}(C)$ and $C / \mathrm{B}(C)$ are complexes consisting of $\mathcal{W}$-Gorenstein modules for any two-degree C-E $\mathcal{W}$ Gorenstein complex $C$. Here we have the following result by Theorem 3.10 and Corollary 4.7.

Corollary 4.9. Let $C$ be a complex. Then the following statements are equivalent:

(1) $C$ is a two-degree $\mathrm{C}$-E $\mathcal{W}$-Gorenstein complex;

(2) $C, \mathrm{Z}(C), \mathrm{B}(C), \mathrm{H}(C), C / \mathrm{Z}(C)$ and $C / \mathrm{B}(C)$ are complexes consisting of two-degree $\mathcal{W}$-Gorenstein modules.

Let $R$ and $S$ be rings. Following [18], an $(S, R)$-bimodule $C={ }_{S} C_{R}$ is semidualizing if:

(1) ${ }_{S} C$ admits a degreewise finite $S$-projective resolution.

(2) $C_{R}$ admits a degreewise finite $R$-projective resolution.

(3) The homothety map ${ }_{S} S_{S} \rightarrow \operatorname{Hom}_{R}(C, C)$ is an isomorphism.

(4) The homothety map ${ }_{R} R_{R} \rightarrow \operatorname{Hom}_{S}(C, C)$ is an isomorphism.

(5) $\operatorname{Ext}_{S}^{\geq 1}(C, C)=0$.

(6) $\operatorname{Ext}_{R}^{\geq 1}(C, C)=0$. 
A left $S$-module $M$ is $C$-projective if it has the form $C \otimes_{R} P$ for some projective left $R$-module $P$. A left $R$-module $N$ is $C$-injective if it has the form $\operatorname{Hom}_{S}(C, E)$ for some injective left $S$-module $E$. Let $\mathcal{P}_{C}=\left\{C \otimes_{R} P \mid P\right.$ is a projective left $R$-module $\}$ and $\mathcal{I}_{C}=\left\{\operatorname{Hom}_{R}(C, E) \mid E\right.$ is an injective left $S$-module $\}$ denote the class of $C$-projective and $C$-injective modules, respectively. Then $\mathcal{P}_{C}$ and $\mathcal{I}_{C}$ are self-orthogonal [13, Corollary 3.2].

If we take $\mathcal{W}=\mathcal{P}_{C}\left(\mathcal{W}=\mathcal{I}_{C}\right)$, then a C-E $\mathcal{W}$ complex is particularly called C-E $C$-projective (C-E $C$-injective) and a two-degree C-E $\mathcal{W}$-Gorenstein complex is particularly called two-degree C-E $C$-Gorenstein projective (C-E $C$-Gorenstein injective). Then we have the following result by Theorem 3.10.

Proposition 4.10. A complex $G$ is two-degree C-E C-Gorenstein projective (two-degree $\mathrm{C}$-E C-Gorenstein injective) if and only if $G$ is $\mathrm{C}-\mathrm{E} C$-Gorenstein projective (C-E C-Gorenstein injective).

$C$-Gorenstein projective modules and $C$-Gorenstein injective modules are introduced and studied in [13], which is different from those defined in [24] ([19]) when $S=R$ is a commutative (Noetherian) ring. If we take $\mathcal{W}=\mathcal{P}_{C}$, then twodegree $\mathcal{W}$-Gorenstein modules are particularly called two-degree $C$-Gorenstein projective modules. We get the following result by Theorem 3.10 and Corollary 4.7.

COROLlary 4.11. Let $R$ and $S$ be rings, ${ }_{S} C_{R}$ be a semidualizing bimodule. Then a left $S$-module $M$ is two-degree $C$-Gorenstein projective if and ony if $M$ is $C$-Gorenstein projective.

If $R$ and $S$ are right and left Noetherian rings respectively and ${ }_{S} C_{R}$ is a dualizing bimodule, then (two-degree) $C$-Gorenstein projective modules are just (two-degree) $V$-Gorenstein projective $(V=C)[10]$; if $R=S$ is a local CohenMacaulay ring admitting a dualizing module $C$, then (two-degree) $C$-Gorenstein projective modules coincide with (two-degree) $\Omega$-Gorenstein projective modules $(\Omega=C)[9]$.

By Corollary 4.11, we obtain the following results.

COROLlary 4.12. Let $R$ be a commutative Noetherian ring. Then an $R$ module $M$ is two-degree $V$-Gorenstein projective if and only if $M$ is $V$-Gorenstein projective.

COROLlary 4.13. Let $R$ be a commutative local Cohen-Macaulay ring admitting a dualizing module $C$. Then an $R$-module $M$ is two-degree $\Omega$-Gorenstein projective if and only if $M$ is $\Omega$-Gorenstein projective.

Remark 4.14. Dually, the results above concerning with $C$-Gorenstein injectivity, $V$-Gorenstein injectivity, $\Omega$-Gorenstein injectivity and Gorenstein injectivity hold. 


\section{REFERENCES}

[1] M. Auslander and M. Bridger, Stable module theory, Mem. Amer. Math. Soc. 94, Amer. Math. Soc., Providence, RI, 1969.

[2] A. A. Beilinson, J. Bernstein and P. Deligne, Perverse sheaves, Analysis and topology on singular spaces, I, Luminy, 1981, Astrisque 100, 1982, 5-171.

[3] A. Beligiannis, Relative homological algebra and purity in triangulated categories, J. Algebra 227 (2000), 268-361.

[4] H. Cartan and S. Eilenberg, Homological algebra, Princeton University Press, 1956.

[5] L. W. Christensen, Gorenstein dimensions, Lecture notes in math. 1747, Berlin, Springer, 2000.

[6] L. W. Christensen And S. Iyengar, Gorenstein dimension of modules over homomorphisms, J. Pure App. Algebra 208 (2007), 177-188.

[7] E. E. Enochs, Cartan-Eilenberg complexes and resolutions, J. Algebra 342 (2011), 16-39.

[8] E. E. Enochs And O. M. G. JENDA, Gorenstein injective and Gorenstein projective modules, Math. Z. 220 (1995), 611-633.

[9] E. E. Enochs And O. M. G. Jenda, Relative homological algebra, Walter de Gruyter, 2000.

[10] E. E. Enochs, O. M. G. Jenda AND J. A. López-Ramos, Dualizing modules and n-perfect rings, Proc. Edinb. Math. Soc. 48 (2005), 75-90.

[11] E. E. Enochs, O. M. G. Jenda And J. XU, Foxby duality and Gorenstein injective and projective modules, Trans. Amer. Math. Soc. 348 (1996), 3223-3234.

[12] J. R. García Rozas, Covers and envelopes in the category of complexes, Chapman \& Hall/ CRC, Boca Raton, FL, 1999.

[13] Y. X. Geng and N. Q. Ding, $\mathcal{W}$-Gorenstein modules, J. Algebra 325 (2011), 132-146.

[14] J. Gillespie, The flat model structure on $\mathrm{Ch}(\mathrm{R})$, Trans. Amer. Math. Soc. 356 (2004), 3369-3390.

[15] R. Hartshorne, Residues and duality, Lecture notes of a seminar on the work of A. Grothendieck, given at Harvard 1963/64, Lecture notes in math. 20, Springer-Verlag, Berlin, 1966.

[16] H. Holm, Gorenstein homological dimensions, J. Pure Appl. Algebra 189 (2004), 167-193.

[17] B. Iversen, Octahedra and braids, Bull. Soc. Math. France 114 (1986), 197-213.

[18] H. Holm AND D. White, Foxby equivalence over associative rings, J. Math. Kyoto Univ. 47 (2007), 781-808.

[19] H. Holm AND P. JørGensen, Semi-dualizing modules and related Gorenstein homological dimensions, J. Pure Appl. Algebra 205 (2006), 423-445.

[20] M. T. Hughes, D. A. Jorgensen And L. M. SEga, Acyclic complexes of finitely generated free modules over local rings, Math. Scand. 105 (2009), 85-98.

[21] F. KASCH, Modules and rings, London. Math. Soc. Monogr. 17, Academic Press, London, 1981.

[22] L. Liang, On $\#-\mathcal{F}$ complexes, Ph.D. thesis, Nanjing University, China, 2011.

[23] S. Sather-Wagstaff, T. Sharif and D. White, Stability of Gorenstein categories, J. Lond. Math. Soc. 77 (2008), 481-502.

[24] D. WhITE, Gorenstein projective dimension with repect to a semidualizing module, J. Commut. Algebra 2 (2010), 111-137.

Bo $\mathrm{Lu}$

College of Mathematics and Computer Science

NORTHWEST UNIVERSITY FOR NATIONALITIES

LANZHOU 730124, GANSU

P.R. CHINA 
Department of Mathematics

NORTHWEST NORMAL UNIVERSITY

LANZHOU 730070, GANSU

P.R. CHINA

E-mail: lubo55@126.com

Wei Ren

Department of Mathematics

NorthWest Normal UniVERSITY

LANZHOU 730070, GANSU

P.R. CHINA

E-mail: rwemail@163.com

Zhongkui Liu

DePARTMENT OF MATHEMATICS

NORThWEST NoRmaL UNIVERSITY

LANZHOU 730070, GANSU

P.R. ChINA

E-mail: liuzk@nwnu.edu.cn 